\title{
Elastic back-scattering patterns via particle surface roughness and orientation from single trapped airborne aerosol particles
}

\author{
Richard Fu, ${ }_{1}^{1}$ Chuji Wang, ${ }^{2}$ Olga Muñoz, ${ }^{3}$ Gorden Videen, ${ }^{1}$ Joshua L. Santarpia, ${ }^{4}$ Yong-Le Pan ${ }^{1, *}$ \\ ${ }^{1}$ Army Research Laboratory, 2800 Powder Mill Road, Adelphi, Maryland 20783, USA \\ ${ }^{2}$ Department of Physics and Astronomy, Mississippi State University, Starkville, Mississippi 39759, \\ USA \\ ${ }^{3}$ Instituto de Astrofisica de Andalucía, CSIC, Glorieta de la Astronomía s/n, 18008, Spain \\ ${ }^{4}$ Sandia National Laboratories, Albuquerque, New Mexico 87123, USA \\ *Corresponding author: yongle.pan.civ@mail.mil
}

We demonstrate a method for simultaneously measuring the back-scattering patterns and images of single laser-trapped airborne aerosol particles. This arrangement allows us to observe how the backscattering patterns change with particle size, shape, surface roughness, orientation, etc. The recoded scattering patterns cover the angular ranges of $\theta=167.7 \circ-180^{\circ}$ including at $180^{\circ}$ exactly and $\phi=0 \circ-360^{\circ}$ in spherical coordinates. The patterns show that the width of the average speckle intensity islands or rings is inversely proportional to particle size and how the shape of these intensity rings or islands also depends on the surface roughness. For an irregularly shaped particle with substantial roughness, the back-scattering patterns are formed with speckle intensity islands, the size and orientations of these islands depend more on the overall particle size and orientation, but have less relevance to the fine alteration of the surface structure and shapes. The back-scattering intensity at $180^{\circ}$ is very sensitive to the particle parameters. It can change from a maximum to a minimum with a change of $0.1 \%$ in particle size or refractive index. The method has potential use in characterizing airborne aerosol particles, and may be used to provide back-scattering information for LIDAR applications.

\section{Introduction}

Elastic light scattering is sensitive to aerosol particle size, shape, complex refractive index, surface roughness, and the molecular density distribution within the particle. Because of its sensitivity, it is considered to be a potential real-time, in situ aerosol classifier or identifier, especially in monitoring lifethreatening bioaerosols from normal atmospheric background constituents [e.g., 1-10]. Although there are various instruments based on elastic scattering that can determine the size, concentration, and asymmetry of particles, the rich information obtained from elastic scattering has not been fully utilized. To retrieve the parameters of a particle from its scattering pattern remains a challenge to the community. The advancement of computational capabilities and improved theoretical models have greatly improved numerical simulations, making it possible to calculate the light scattering from highly irregular, heterogeneous systems [e.g. 11-18]. There remains a demand for experimental data from single particles that can be used for verification of theoretical calculations and help develop more accurate algorithms. One of the approaches is to trap single particles for detailed measurements such as obtaining the angular distribution of light-scattering intensity from a single trapped biological cell in liquid or from a trapped airborne droplet [e.g., 19-23]. A stable trap can provide time-resolved dynamic measurements of particle properties in time as conditions change, supply more accurate scattering angle assignment and control the particle orientation that is very hard for flowing through systems [e.g. 3-10]. There are currently limited publications of scattering patterns from single trapped solid airborne particles, especially in the exact and near back-scattering directions [24]. It is not trivial to obtain the scattering signals from a single micronsize particle at the exact backscattering polar angle $\theta=180^{\circ}$, since stray scattered light from optical 
components can be significant [24-25]. Whereas, the forward-scattering region is determined largely by diffraction and is sensitive to particle morphology, the back-scattering region is sensitive especially to particle heterogeneities and surface structure, including surface roughness [26]. Many real applications operate in a LIDAR configuration, in which the exact back-scattering signal is analyzed for information.

Scattering patterns from a single complex particle with rough surface always appear with specklelike islands, it is very difficult to retrieve particle parameters even just the size by comparing these patterns with theory. However, particle size can be approximately retrieved from the dependence of scattering intensity on the polar scattering angle $\theta$. Such an intensity variation depends more strongly on the particle size than on the refractive index [27]. The frequency of the scattering intensity oscillation can be obtained through Fourier transformation, autocorrelation analysis or other pattern analysis algorithms and subsequently related to particle size $[8,9,27]$. Information about particle shape and orientation is more concentrated in the intensity variation along the azimuthal scattering angle $(\phi)[8,9,27]$. It is found that the median surface area of intensity peaks (the size speckle islands) is inversely proportional to particle size, especially in the near forward scattering region. This trend holds well for most particle types with substantial roughness or complexity [27-29].

In this paper, we investigated the sensitivity of the particle size, shape, surface roughness, or orientation on the back-scattering patterns. We designed and assembled an apparatus that can measure the back-scattering patterns and images of a single laser-trapped particle simultaneously. We trap both transparent and absorbing particles with arbitrary morphology using a recently developed trapping technology via a single shaped laser beam [30]. The recorded scattering patterns cover the range of $\theta=167.7 \circ-180^{\circ}$ including at $180^{\circ}$ exactly and $\phi=0 \circ-360^{\circ}$ in spherical coordinates. Here $\theta$ is the polar angle relative to the $\mathrm{z}$ axis that is defined by the direction of the incident laser beam, and $\phi$ is the azimuthal angle relative to some arbitrarily determined $\mathrm{x}$ axis, in this case, perpendicular to the laboratory floor.

\section{Experimental arrangement}

A schematic of the experimental arrangement with the corresponding coordinates is shown in Fig. 1. A power-adjustable $532 \mathrm{~nm}$ laser (up to $3 \mathrm{~W}$ ) is used to trap airborne particles. The laser beam is spatially filtered (L6, PH2, L7) and then formed into a collimated hollow beam after passing two axicons (AL1, AL2). The hollow beam is reflected vertically upward by a reflective mirror $(\mathrm{M})$ and then focused by an aspheric lens (ASL3, numerical aperture $\mathrm{NA}=0.60$; effective focal length efl $=12 \mathrm{~mm}$ ) to create an upward hollow optical cone with a focal point at the vertex. Particles can be trapped in air at or near the focal point depending on the particle properties [30]. All tested aerosol particles are introduced into the trapping chamber by sprinkling them using a cotton-tipped swab through a hole in the top of the enclosed chamber. This hole is an adjustable iris that can be closed when particles are delivered into the chamber. The chamber is used to minimize the perturbations caused by air flow in the lab. The trapped particle is illuminated by a circularly polarized $465 \mathrm{~nm}$ laser beam propagating horizontally (defined to be along the z-axis). This beam is spatially filtered and collimated (L1, PH1, L2) before passing through a polarizing beam splitter (PBS1) and a quarter-wave plate $(\lambda / 4 \mathrm{WP})$ to circularly polarize the incident beam. It is lightly focused (L3) before being reflected by a non-polarized beam splitter (BS1) toward the trapped particle. The back-scattered light is transmitted through the same beam splitter (BS1) and then collected by lens ASL2 (efl $=50$ $\mathrm{mm})$. ASL2 is aligned so that the trapped particle is in the back focal point of the lens. A ray emerging from the focal point and entering the lens (focal length $=f$ ) at an angle $\theta$ and emerging parallel to the optical axis (-z direction) of the lens with an off-axis displacement $r$ satisfies the Abbe sine condition $r=f \sin \theta$. The collected scattering light via ASL2 is further separated by another non-polarized beam splitter (BS2). Half of the light is recorded by an image-intensified charge-coupled device (ICCD1) after a short pass filter (SP, cutoff at $500 \mathrm{~nm}$ ). The other half of the scattered light is imaged on to another detector (ICCD2) after passing through a band pass 
filter (BP, band pass for the $532 \mathrm{~nm}$ scattering light). The two ICCDs are Andor Istar cameras with the same specifications, and both are run in an external trigger mode. They record the pattern and image simultaneously and are activated only when a trigger pulse is received from a pulse generator running at $2 \mathrm{~Hz}$. The intensifiers are gated at $200 \mu \mathrm{s}$ and $50 \mu \mathrm{s}$ for recording the scattering pattern and image, respectively; therefore, every recorded scattering pattern has an associated image of the trapped particle at the same moment. Through the sine condition, the $\mathrm{x}$ and $\mathrm{y}$ coordinates of the recorded pattern on the camera ICCD1 can be traced back to the scattering angles $\theta, \phi$ as shown in the inset on the middle left of Fig. 1. ICCD2 records the image at an equivalent resolution of $0.6 \mu \mathrm{m} /$ pixel. An additional CCD is used to capture the shadow image of the trapped particle illuminated by a white light-emitting diode (LED). These shadow images give the side view of the particle, although they cannot be precisely synchronized frame by frame with the images obtained by ICCD2, but at least are captured within one second of the other images.

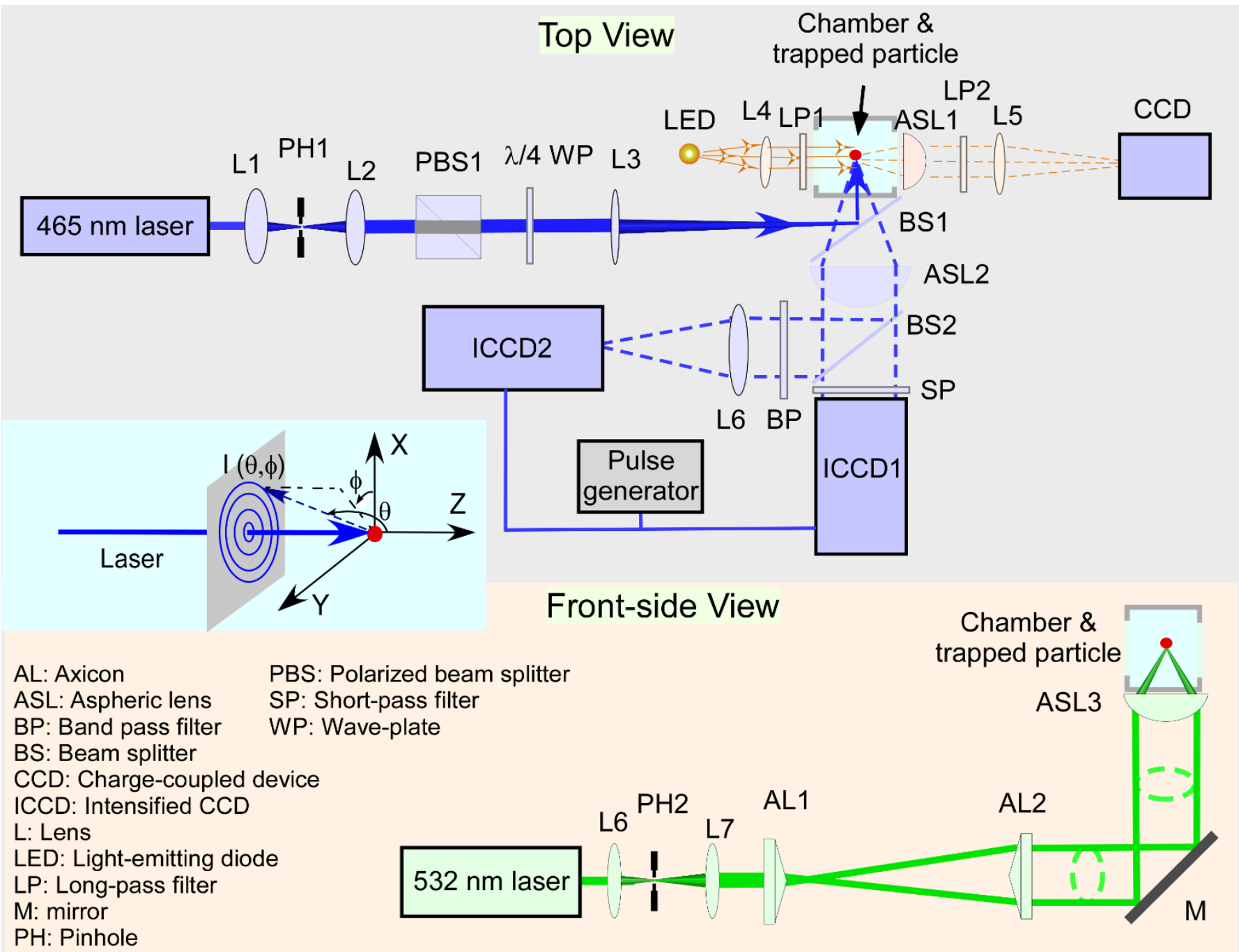

Fig. 1. Experimental setup for measuring elastic back-scattering patterns and images simultaneously from single, laser-trapped airborne aerosol particles. The inset shows the coordinate system, and the legend of acronyms is in the lower left.

The aerosol particles we examined include NIST-traceable polystyrene microsphere (Duke Scientific 4210A, diameter $d=10.0 \mu \mathrm{m} \pm 0.05 \mu \mathrm{m}$, index of refraction $n=1.59 @ 589 \mathrm{~nm}$ ); NIST-traceable 
Borosilicate glass microspheres (Duke Scientific 9010, diameter $d=10.0 \mu \mathrm{m} \pm 1.0 \mu \mathrm{m}$, index of refraction $n=1.56 @ 589 \mathrm{~nm})$; Johnson grass spores (6.2-9.8 $\mu \mathrm{m}$, Greer); and volcanic ash collected from Eyjafjallajökull [31]. These ash particles were collected directly from a surface deposit shortly after the eruption that occurred in April 2010 about $5 \mathrm{~km}$ from the source. The majority of the particles were composed of silicates with sizes ranging from submicron to tens of microns. Measured refractive indexes were ranged from 1.43 to 1.59 for the real part and from non-absorbing to 0.004 for the imaginary part [32-33].

Absorbing particles are trapped mainly by the photophoretic force [30], and the laser power used for trapping the Johnson grass spores and volcanic ash was $100 \mathrm{~mW}$. The non-absorbing particles are trapped by radiative pressure force [30], and the laser power used for trapping the polystyrene and glass microspheres was $1.4 \mathrm{~W}$. Once a particle is captured, it can be trapped stably in air for hours even using a much lower trapping laser power of a few $\mathrm{mW}$. In some cases, a trapped particle could oscillate around a point with a displacement up to tens of microns or rotate or spin along an axis near the trapped position. In addition, the $465 \mathrm{~nm}$ illumination laser used for generating the light-scattering patterns also contributes to the photophoretic and radiation pressure forces for trapping. Its power was kept as low as $18 \mathrm{~mW}$ to minimize its influence on particle trapping. The trapping positions are slightly different, but within a few micron dislocation for particles with different size, shape, and absorption coefficients. They are moved to the same location by finely tuning the trapping laser power and beam position. Their positions can be determined by the pixel position of the particle images from the side view CCD and the back view ICCD2.

\section{Results and discussion}

In order to examine the light-scattering sensitivity to the particle surface roughness, we selected three approximately spherical particles with different surface roughness and one irregularly shaped particle for the study. These particles are a polystyrene microsphere, a Borosilicate glass microsphere, a Johnson grass spore, and a volcanic ash particle. These are all sized around $10 \mu \mathrm{m}$. The top row in Fig. 2 shows typical back-scattering patterns obtained from one of each single trapped particles recorded by ICCD1 with their associated images (middle row) recorded by ICCD2. From the SEM images of these particles shown in the bottom row, we notice that polystyrene particle looks like a perfect sphere with a very smooth surface, and the surfaces appear coarser as we continue to the right, going from polystyrene to glass microsphere to Johnson grass spore. The trapped volcanic ash particles have highly irregular shapes far from a sphere.

For spherically symmetric particles, the resulting light scattering consists of concentric rings. As the symmetry breaks due to heterogeneities or surface roughness, these rings become broken and distorted. The amount of change in the pattern depends on the region. In the forward direction, the light scattering is governed largely by diffraction, which is less sensitive to these changes than the backscattering region. For the polystyrene and glass microspheres, we see only minor variations from the concentric rings. Johnson grass spores have an inhomogeneous density distribution and much rougher surfaces but still largely retain a spherical shape. The back scattering patterns still retain evidence of the concentric ring structure, but there are significant intensity variations, and the rings appear to be composed of large speckles. In the very center of the pattern, we can see that the ring structure disappeared and left with speckle islands of higher intensity. It appears that all the three spherical particles contain 4 high intensity rings along the polar scattering angle within the range of $\theta=167.7 \circ-180^{\circ}$, a

result of their having similar particle size, regardless of the difference of their refractive index and surface roughness; i.e., the oscillation frequency of scattering intensity along the polar scattering angle still appears to be dominated by the overall particle size, while the particle surface roughness contributes to strong intensity deviations along the azimuthal scattering angle. Once the particle shape is far from a sphere, no more concentric rings remain as demonstrated by the volcano ash particle. In this case, there is 
no periodic intensity oscillation along the polar scattering direction. However, the average widths of these speckle intensity islands remain comparable to the size of the concentric rings from the similar size microsphere. Similar to the forward scattering, this suggests that the width of the speckle intensity islands or rings is inversely proportional to a scatterer's size.
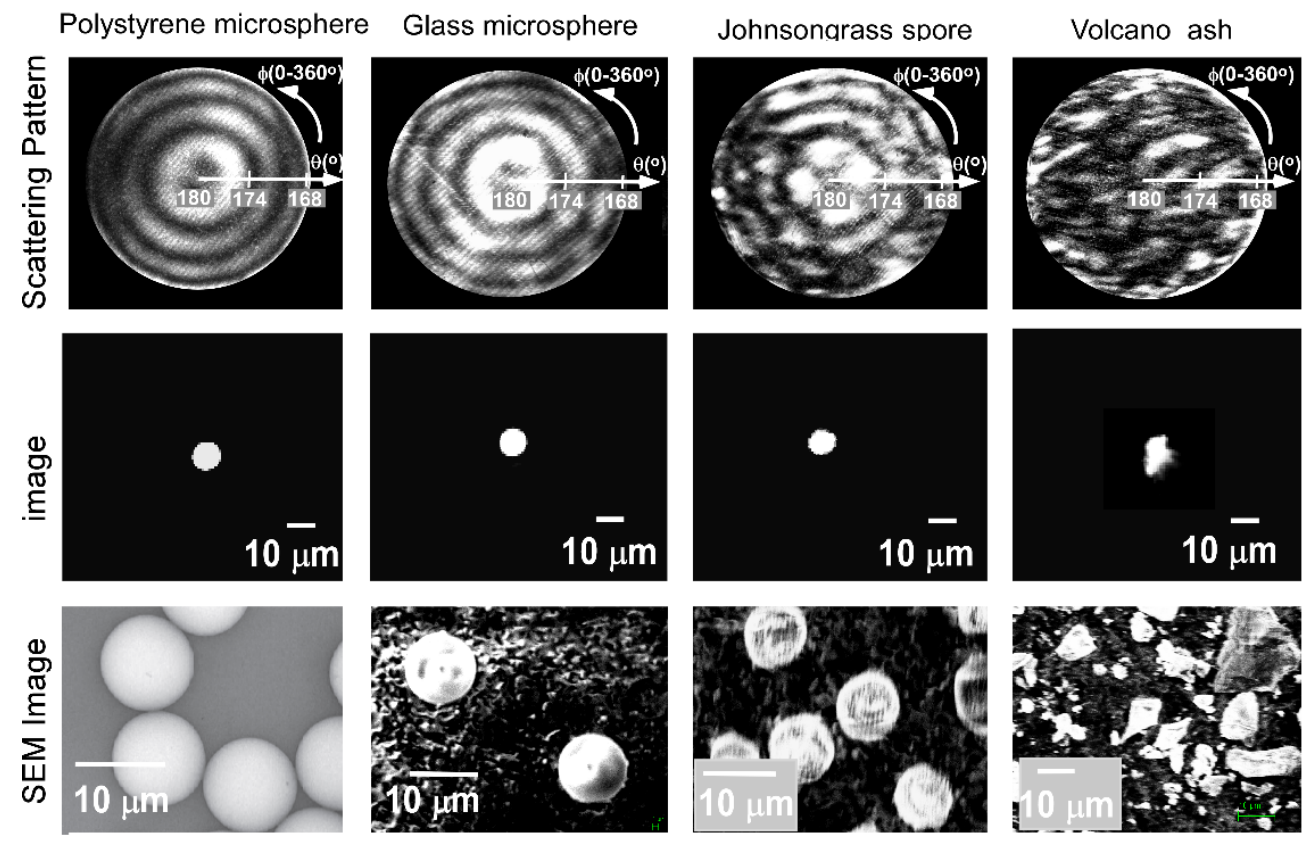

Fig. 2. Typical back-scattering patterns (top row) obtained from laser-trapped aerosol particles (recorded by ICCD1), their associated images (middle row, recorded by ICCD2) when the scattering patterns were recorded, and SEM images of the corresponding aerosol particles in each column (bottom row). The particles in the four columns are a polystyrene microsphere, a Borosilicate glass microsphere, a Johnson grass spore, and a volcanic-ash particle, respectively.

Fig. 3 shows the comparison of the experimental measurements with the Lorenz-Mie simulations [34] for single individual trapped microspheres in air. The Lorenz-Mie simulations are obtained using a microsphere with (a) a refractive index $n=1.60975+\mathrm{i}$, and radii of $r=5.104 \mu \mathrm{m} ; 5.109 \mu \mathrm{m} ; 5.114 \mu \mathrm{m} ; 5.119 \mu \mathrm{m}$; and $5.124 \mu \mathrm{m}$ for the polystyrene microsphere; (b) $n=1.567+\mathrm{i} 0$, and $r=5.050 \mu \mathrm{m} ; 5.055 \mu \mathrm{m} ; 5.0625$ $\mu \mathrm{m} ; 5.065 \mu \mathrm{m}$; and $5.070 \mu \mathrm{m}$ for the Borosilicate glass microsphere; and (c) $r=4.50 \mu \mathrm{m}$; $n=1.510+\mathrm{i} 0.0015 ; 1.515+\mathrm{i} 0.0015 ; 1.520+\mathrm{i} 0.0015 ; 1.525+\mathrm{i} 0.0015$; and $1.530+\mathrm{i} 0.0015$ for the Johnson grass spore. Refractive index $n$ is obtained from Polyanskiy [35] for polystyrene and Borosilicate glass. For the bacterial spores and vegetative cells, its value is estimated to be in a range of 1.510-1.525 for the real part and 0.0015 for the imaginary part [36]. The experimental curves (dark $\times$ ) shown in Fig. 3 (a-d) are obtained by averaging over all angles $\phi$ from the corresponding patterns shown in Fig. 2 (a-d). For a perfect microsphere, the scattering intensity should be independent of $\phi$ when under illumination of a circularly polarized laser. However, variations of the intensity along $\phi$ measured here demonstrate that none of these particles is perfectly spherical.

We were unable to obtain a perfect match of the Lorenz-Mie simulations with the measured data for any of the particles we examined. Minor discrepancies between the experimental and the theoretical data for the intensity distribution along angle $\theta$ are mostly due to particle nonsphericity although other 
factors do come into play, like misalignment and beam imperfections. Although the positions and number of maxima show little difference in the observed scattering region (167.7०-180\%) as the Lorenz-Mie parameters change within their narrow limits (Fig. 3), the light-intensity can change dramatically, even from a minimum to a maximum at $180^{\circ}$ with a $0.1 \%$ change in particle size $(5 \mathrm{~nm})$ or refractive index (0.005). In the anti-solar direction, strong scattering pattern of concentric, colored rings near a shadow can be frequently observed in nature with clouds of mist, such an optical phenomenon is called glory [37]. We also can see that the glory that occurs near $180^{\circ}$ is not always a strong phenomenon at $180^{\circ}$, the wellknown back-scattering peak may be significantly dampened due to the strong angular resonances occurring in spherical particles. Such a strong sensitivity of the back-scattering intensity at $180^{\circ}$ is relevant in assessing aerosol metrology using LIDAR. The light-scattering patterns of the PSL microsphere are captured in two different times. We can see that in one case (red + ), the pattern resembles more closely an $r=5.114 \mu \mathrm{m}$ sphere, with a relatively strong scattering intensity at $180^{\circ}$. In the other case (black $\times$ ), the pattern more closely resembles an $r=5.119 \mu \mathrm{m}$ sphere, with a weak scattering intensity at $180^{\circ}$ and stronger maximum peak around $177^{\circ}$. This suggests an aspect ratio on the order of 0.998 can be measured easily from the back-scattering signal.
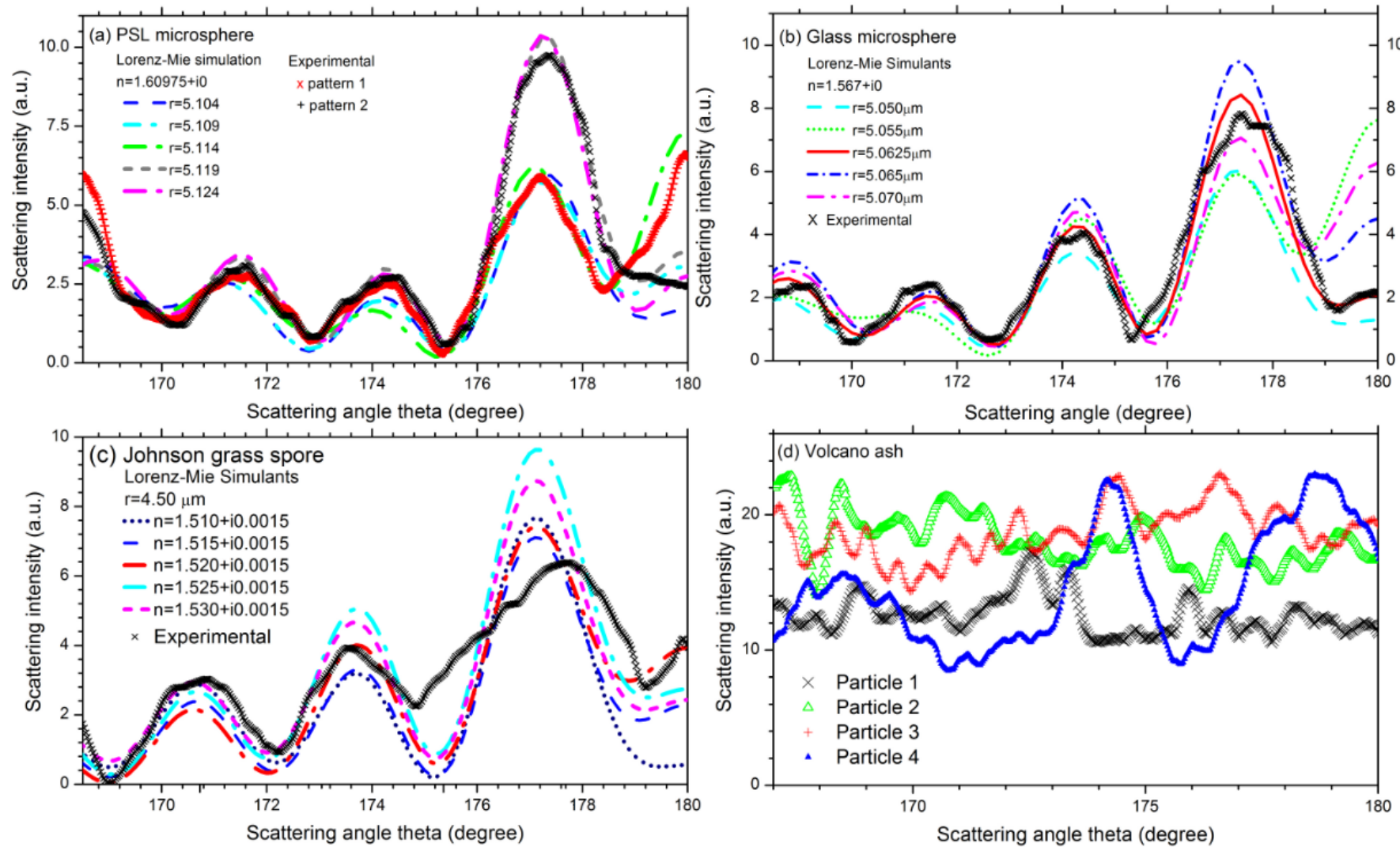

Fig. 3. Azimuthally averaged light-scattering intensities of a single, laser-trapped (a) polystyrene microsphere, (b) Borosilicate glass microsphere, (c) Johnson grass spore, and (d) a volcanic ash particle. The plots show the LorenzMie calculations from a microsphere having parameters similar to the measured particles $(\mathrm{a}, \mathrm{b}, \mathrm{c})$ for comparison.

The scattering patterns of the irregularly shaped Johnson grass spore deviate significantly from that of a sphere. However, the calculations can still be used to obtain a rough estimation of the particle parameters by considering peak numbers and positions. For the highly irregular volcano ash particles, the scattering intensities do not present distinctive peaks and we also do not see an obvious glory as we increase the scattering angle toward 
$180^{\circ}$. The curves shown in Fig 3 (d) are from four particles with the longest dimensions of $15 \mu \mathrm{m}$ (whose scattering pattern is shown in Fig 2), $30 \mu \mathrm{m}$ (whose scattering pattern is shown in Fig 4), $25 \mu \mathrm{m}$, and $12 \mu \mathrm{m}$. The process of azimuthally averaging significantly reduces the magnitudes of the intensity peaks. However, the patterns do suggest that the number of peaks in these patterns is still proportional to particle size.

Fig. 4 shows pairs of back-scattering patterns obtained from the same trapped aerosol particles consisting of a single polystyrene microsphere, two attached Johnson grass spores, and an aggregate of volcanic ash particles in different orientations recorded at different times. The scattering patterns for the polystyrene microsphere in Fig 4 and media 1 are from the same trapped particle shown in Fig. 2a. Media 1 shows that the first 9 patterns are almost identical (presented as the upper pattern). The remaining patterns are different but similar to themselves (presented in the bottom row). During the two second period between image acquisitions, the particle system might change slightly, either through a particle rotation that makes the PSL microsphere face the illumination laser at different orientations, as the PSL might not be a perfect sphere with an ultra-small non-sphericity, or through contamination either by dust or moisture as the air in the lab can flow freely into and out from the chamber. While such small changes are not visible in the images, they can be seen in the light-scattering patterns. Even the patterns from the nearly spherical polystyrene microsphere show significant asymmetries are present.

\section{Polystyrene microsphere Two Johnsongrass spores Aggregates of Volcano ashes}
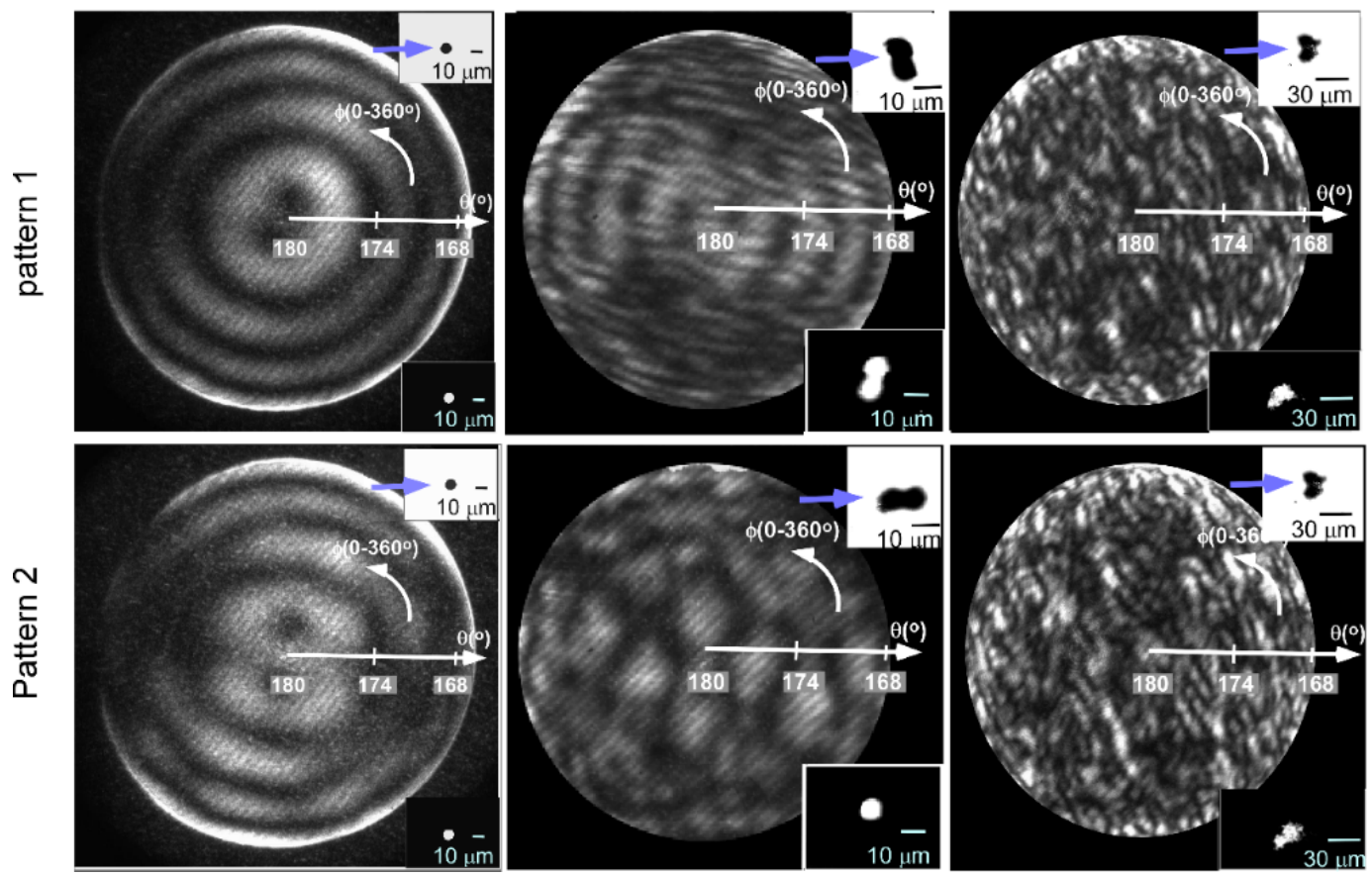

Fig. 4. Two back-scattering patterns obtained from the same trapped aerosol particles. Particles consist of a single polystyrene microsphere (left column), two attached Johnson grass spores (middle column), and an aggregate of volcanic ash particles (right column) in different orientations, recorded at different times. The upper right corner of each frame shows a side-view shadow image of the trapped particle. The arrow indicates the laser illuminating direction. The bottom right corner of each frame shows a rear-view image of the trapped particle. Media 1 (MEDIA 1 FILE: "media1-PSLTAOS_IMAGE.wmv"), 2 (MEDIA 2 FILE: "media2-2BJTAOS_IMAGE.wmv"), and 3 (MEDIA 3 FILE: "media3-VASH_TAOS-Image.wmv") show 20 successive back-scattering patterns recorded with ICCD1 and their associated rear-view images recorded with ICCD2 in a 10 second time interval ( 0.5 second time separation between two of the successive patterns and images) for the three corresponding trapped particles. 


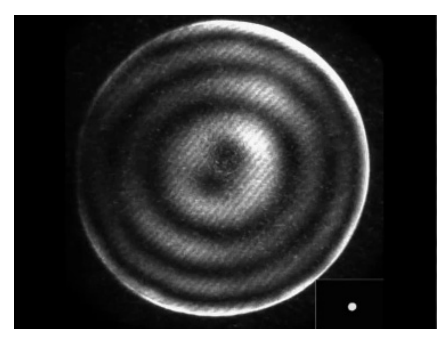

Media 1

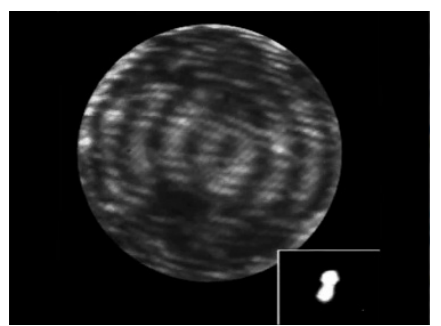

Media 2

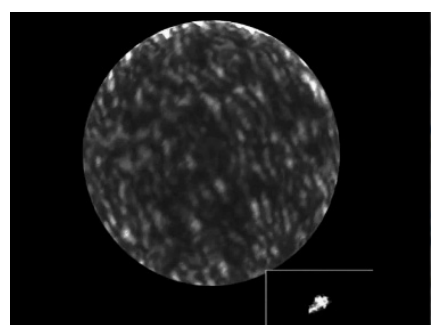

Media 3

(media1-PSLTAOS_IMAGE.mp4) (media2-2BJTAOS_IMAGE.mp4) (media3-VASH_TAOS-Image.mp4)

The light-scattering pattern from the two attached Johnson grass spores that is vertically orientated (top middle panel) suggests an interference pattern from two scattering centers, with a horizontal band of high intensity through the center. Such a pattern is similar to what is obtained from two attached single spheres to which this system resembles $[15,16]$. The spatial frequency of the intensity oscillations in the vertical direction is approximately twice that of the horizontal direction, which is what we would expect from a particle whose extent is half as great in this direction. The scattering pattern in the end-on illumination (lower middle pattern) is similar to that of the single Johnson grass spore of Fig. 2: we see a similar frequency of oscillations, but it also shows greater azimuthal variations in the patterns for the end-on spores than for the single spore.

The scattering patterns from the single aggregate of volcanic ashes show a speckle pattern with islands of high intensity. In some patterns the speckle appears uniformly distributed and in other patterns, there are patches where the speckle is of higher intensity. The latter occurs more frequently in the lower pattern, for instance. While the characteristics of the patterns is largely dependent on particle size, quantification of the speckle locations and shapes changes significantly with small changes in orientation. The number and frequency of high-intensity regions located within the speckle patterns can also change significantly as the particle orientation changes [e.g. 5-6, 26-29].

\section{Summary}

We demonstrated an apparatus that could measure simultaneously the backscattering patterns and images of a single laser-trapped particle. Such a setup allows us to observe how the back-scattering patterns change with a particle size, shape, surface roughness, or orientation, etc. The recorded scattering patterns cover the range of $\theta=167.7^{\circ}-180^{\circ}$ and $\phi=0^{\circ}-360^{\circ}$ in spherical coordinates. The patterns and their corresponding images suggest the width of the average speckle intensity islands or rings is inversely proportional to particle size in the back-scattering region. This is similar to what is observed in the forward-scattering region; however, the forward-scattering signal is dominated by diffraction, which does not exist in the back-scattering direction. For irregularly shaped particles like volcanic ash, the back-scattering patterns appear as speckle-intensity islands. The size and orientation of the islands appears to depend significantly on the overall particle size and orientation. Small changes in surface structure and shape provide finer alterations to the scattering patterns. Further detailed statistical analysis from a large set of such particles having different parameters is currently in preparation.

Funding Defense Threat Reduction Agency (HDTRS1518237, HDTRA1619734); US Army Research Laboratory mission funds.

\section{References}

1. P. J. Wyatt, "Identification of Bacteria by Differential Light Scattering," Nature 221, 1257-1258 (1969). 
2. M. Bartholdi, G. C. Salzman, R. D. Hiebert, and M. Kerker, "Differential light scattering photometer for rapid analysis of single particles in flow," Appl. Opt. 19, 1573-1581 (1980).

3. P. H. Kaye, K. Alexander-Buckley, E. Hirst, and S. Saunders, "A real-time monitoring system for airborne particle shape and size analysis,” J. Geophys. Res. 101, 19215-19221 (1996).

4. R. A. West, L. R. Doose, A. M. Eibl AM, T. M. Tomasko, M. I. Mishchenko, "Laboratory measurements of mineral dust scattering phase function and linear polarization," J. Geophys. Res. 102, 16871-16881 (1997).

5. Y.-L. Pan, K. B. Aptowicz, R. K. Chang, M. Hart, J. D. Eversole, "Characterizing and monitoring respiratory aerosols by light scattering," Opt. Lett. 28, 589-591 (2003).

6. K. B. Aptowicz, R. G. Pinnick, S. C. Hill, Y.-L. Pan, R. K. Chang, "Optical scattering patterns from single urban aerosol particles at Adelphi, Maryland, USA: A classification relating to particle morphologies," J. Geophys. Res. 111, D12212 (2006).

7. X. Cao, G. Roy, and R. Bernier, "Lidar polarization discrimination of bioaerosols," Opt. Eng. 49, 116201 (2010).

8. Y.-L. Pan, M. J. Berg, S. S.M. Zhang, H. Noh, H. Cao, R. K. Chang, and G. Videen, "Measurement and autocorrelation analysis of two-dimensional light-scattering patterns from living cells for label-free classification," Cytometry A 79, 284-292 (2011).

9. K. B. Aptowicz, Y.-L. Pan, S. D. Martin, E. Fernandez, R. K. Chang, and R. G. Pinnick, "Decomposition of atmospheric aerosol phase function by particle size and asphericity from measurements of single particle optical scattering patterns," J. Quant. Spectrosc. Radiat. Transf. 131, 13-23 (2013).

10. V. Sivaprakasam, J. Czege, J. D. Eversole, "Wavelength resolved polarized elastic scatter measurements from micron-sized single particles," Vasanthi Sivaprakasam, Jozsef Czege, Jay D. Eversole, Proc. SPIE 8710, 871018 (2013).

11. M. I. Mishchenko, L. D. Travis, and A. A. Lacis, Scattering, Absorption, and Emission of Light by Small Particles (Cambridge University Press, New York, 2002).

12. C. M. Sorensen, D. Shi, "Patterns in the ripple structure of Mie scattering," J. Opt. Soc. Am. A 19,122$125(2002)$.

13. E. Zubko, Y. Shkuratov, M. Hart, J. Eversole, and G. Videen, "Backscattering and negative polarization of agglomerate particles," Opt. Lett. 28, 1504-1506 (2003).

14. M. J. Berg, C. M. Sorensen, and A. Chakrabarti, "Explanation of the patterns in Mie theory," J. Quant. Spectrosc. Radiat. Transfer 111, 782-794 (2010).

15. G. Videen, W. Sun, Q. Fu, D. R. Secker, R. Greenaway, P. H. Kaye, E. Hirst, D. Bartley, "Light scattering from deformed droplets and droplets with inclusions: II. Theoretical treatment," Appl. Opt. 39, 5031-5039 (2000).

16. D. R. Secker, R. Greenaway, P. H. Kaye, E. Hirst, D. Bartley, G. Videen, "Light scattering from deformed droplets and droplets with inclusions: I. Experimental Results,” Appl. Opt. 39, 5023-5030 (2000).

17. G. Videen, W. Sun, and Q. Fu, "Light scattering from irregular tetrahedral aggregates,” Opt. Comm. 156, 5-9 (1998).

18. O. Muñoz, F. Moreno, D. Guirado, D. D. Dabrowska, H. Volten, J. W. Hovenier, "The AmsterdamGranada Light Scattering Database,’J. Quant. Spectrosc. Radiat. Transfer, 113, 565-574 (2012).

19. R. M. P. Doornbos, M. Schaeffer, A. G. Hoekstra, P. M. A. Sloot, B. G. de Grooth, and J. Greve, "Elastic backscattering measurements of single biological cells in an optical trap," Appl Opt 35 (4), p.729-734 (1996)

20. D. Watson, N. Hagen, J. Diver, P. Marchand, M. Chachisvilis, "Elastic light scattering from single cells: orientational dynamics in optical trap,” Biophysical J., 87 (2), 1298-1306 (2004) 
21. M. D. Barnes, N. Lermer, W. B. Whitten, and J. M. Ramsey, “A CCD based approach to high-precision size and refractive index determination of levitated microdroplets using Fraunhofer diffraction," Rev. Sci. Instrum. 68, 2287-2291 (1997).

22. U. K. Krieger, C. Marcolli and J. P. Reid, "Exploring the complexity of aerosol particle properties and processes using single particle techniques, DOI: 10.1039/C2CS35082C (Critical Review)," Chem. Soc. Rev., 41, 6631-6662 (2012)

23. M. Guillon, K. Dholakia, D. McGloin, "Optical trapping and spectral analysis of aerosols with a supercontiuum laser source,” Opt Express. 16(11),7655-64 (2008).

24. Y.-L. Pan, C. Wang, L. A. Beresnev, A. J. Yuffa, G. Videen, D. Ligon, J. L. Santarpia, "Measurement of back-scattering patterns from single laser trapped aerosol particles in air,", Submitted to Applied Optics

25. B. M. Heffernan, Y. W. Heinson, J. B. Maughan, A. Chakrabarti, and C. M. Sorensen, "Backscattering measurements of micron-sized spherical particles," Appl. Opt. 55, 3214-3218 (2016)

26. G. E. Fernandes, Y.-L. Pan, R. K. Chang, K. Aptowicz, R. G. Pinnick, "Simultaneous forward- and backward-hemisphere elastic-light-scattering patterns of respirable-size aerosols," Opt. Lett. 31, 3034 3036 (2006).

27. Z. Ulanowski, E. Hirst, P. H. Kaye, R. Greenaway, "Retrieving the size of particles with rough and complex surfaces from two-dimensional scattering patterns," J. Quantitative Spectrosc. \& Radiative Transfer, 113 (18), 2457-2464 (2012).

28. S. Holler, Y. L. Pan, R. K. Chang, S. Hill, R. G. Pinnick, and J. R. Bottiger, "Two-dimensional elastic scattering angular patterns for the characterization of airborne micro-particles," Optics Letters, 23 (18), 1489-1491 (1998).

29. S. Holler, S. Zomer, G. F. Crosta, Y. L. Pan, R. K. Chang, and J. R. Bottiger, "Multivariate analysis and classification of two-dimensional angular optical scattering patterns from aggregates," Applied Optics 43 (33), 6198-6206 (2004).

30. B. Redding and Y.-L. Pan, "Optical trap for both transparent and absorbing particles in air using a single shaped laser beam,” Optics Letters 40, 2798-2801 (2015).

31. S. Merikallio, O. Muñoz, A. Sundstrm, T. Virtanen, M. Horttanainee, G. Leeuw and T. Nousiainen, "Optical modeling of volcanic ash particles using ellipsoids", J. Geophysical Research, Vol 120 (9), 4102-4116 (2015)

32. S. M. Newman, L. Clarisse, D. Hurtmans et al., "A case study of observations of volcanic ash from the Eyjafjallajökull eruption: 2. airborne and satellite raddiative measurements," Journal of Geophysical Research: Atmospheres, 117 (D20), (2012).

33. U. Schumann, B.Weinzierl, O. Reitebuch et al., "Airborne observations of the Eyjafjalla Volcano ash cloud over Europe during air space closure in April and May 2010," Atmos. Chem. Phys., 11, 2245-2279 (2011).

34. P. Laven, "MiePlot: A computer program for scattering of light from a sphere using Mie theory \& the Debye series", http://www.philiplaven.com/mieplot.htm

35. M. Polyanskiy, "Refractive Index Database," http://refractiveindex.info/?shelf=glass\&book=HIKARIBK\&page $=$ J-BK7A.

36. K. F. A. Ross and E. Billing, "The water and solid content of living bacterial spores and vegetative cells as indicated by refractive index measurements," J. Gen. Microbiol., 16 418-425 (1957).

37. M. Minnaert, Light and Color in the Outdoors (Springer-Verlag, 1974). 